\title{
NECESIDAD DE FORTALECIMIENTO DE LA FORMACIÓN DOCENTE EN LA ARTICULACIÓN ESCUELA MEDIA-UNIVERSIDAD AL ENSEÑAR CIENCIAS
}

Comentarios al libro de BIOLATTO, Renato (Compilador) y otros. La Enseñanza de las Ciencias. Reflexiones y Propuestas. Editorial Laborde. Rosario - Argentina, 2011; 157 páginas.

Natalia Fátima Sgreccia (Universidad Nacional de RosarioConsejo Nacional de Investigaciones Científicas y Técnicas)*

sgreccia@fceia.unr.edu.ar

Recibido: 29/07/2011 Aceptado: 26/10/2011

En este libro, docentes-investigadores de la Universidad Nacional de Rosario ofrecen un panorama amplio acerca de lo que involucra enseñar ciencias en el nivel secundario de educación en la actualidad.

Al comentar sobre el libro (pp. 17-20), Renato Biolatto (Secretaría de Extensión Universitaria) presenta algunos desafíos vigentes en la articulación Escuela Media - Universidad, entre ellos la alfabetización científica de la población, y comenta que, como acciones concretas en este sentido, se han realizado jornadas y foros durante dos años cuyas conclusiones se constituyeron en base para la elaboración de esta obra. Mediante actividades y materiales de este tipo se apuesta a la constante formación y actualización docente.

María Alejandra Pardal (Facultad de Ciencias Bioquímicas y Farmacéuticas), en el capítulo titulado "Un proceso sostenido de trabajo colaborativo y continuos desafíos. Hechos, escenarios y actores comprometidos en la experiencia" (pp. 21-25), le atribuye a las ciencias un rol protagónico en la construcción de una sociedad justa, equitativa y democrática. Reconoce a ésta

* Magister en Didácticas Específicas. Universidad Nacional del Litoral. Profesora en Matemática. Universidad Nacional de Rosario. Doctoranda en Educación (UNR), con beca del CONICET. 
como meta compartida por todas las propuestas del libro. También identifica como punto de convergencia de la jornada y foros realizados a la necesidad y el desafío de: fortalecer la formación docente inicial y continua, revisar y repensar los diseños curriculares, y mejorar la articulación entre los distintos niveles del sistema educativo.

María Cristina Lesquiuta, Liliana Boccardo y Renato Biolatto (Secretaría de Extensión Universitaria), en el capítulo titulado "De tramas, redes y puentes" (pp. 27-40), expresan que la extensión, junto a la docencia, investigación y gestión, constituye uno de los pilares de la acción universitaria. Una de las problemáticas que compete al área de articulación es el espacio transicional entre el nivel medio y superior, contemplando una preocupante deserción anual que ronda el $40 \%$ en la Universidad Nacional de Rosario. Consideran a tal fenómeno multicausal, por lo que han venido desarrollando distintas líneas de acción: espacios de trabajo entre docentes de ambos niveles educativos, instancias de perfeccionamiento a docentes del nivel medio y propuestas de tutorías como transversales a las prácticas curriculares. Mencionan los principales avances de la jornada y foros realizados, subrayando como ejes de reflexión a: la generación de condiciones de enseñanza de las ciencias, la elaboración de problemáticas y saberes para ser enseñados, así como la recuperación de escenas cotidianas desde basamentos científicos.

Gloria Rossi (Facultad de Psicología), en el capítulo titulado "La(s) adolescencia(s) hoy. Problemáticas y desafíos en la producción de proyectos identificatorios y las elecciones académicas, en un contexto de incertidumbre social" (pp. 41-55), reconoce doce dimensiones de análisis que considera fundamentales para la transición nivel medio-universidad en la actualidad: los ideales que comandan las elecciones académicas en cada sujeto, las posiciones en relación a los ideales intergeneracionales y parentales, la búsqueda de una identidad profesional, decisiones acerca del proyecto identificatorio de la persona, las posiciones subjetivas frente a la tramitación de la herencia cultural respecto de la producción de un proyecto académico, las representaciones sociales imaginarias construidas, el atravesamiento de un doble modelo de transmisión: cofigurativo y postfigurativo, la crisis sociopolítica y la transmutación cultural, el lugar del trabajo como referente para la construcción de las identidades, la pretendida libertad e igualdad de los actores sociales ante el mercado, la vivencia subjetiva individual y colectiva de inseguridad, y la prevalencia de la incertidumbre en los tiempos actuales. Ante esta sociedad, que considera de riesgo, visualiza a los graduados universitarios como posibles agentes de cambio.

Enrique Barés (Facultad de Humanidades y Artes, Facultad de Psicología), en el capítulo titulado "Educación y conocimientos en la relación sociedad / estado / escuela / universidad" (pp. 57-76), realiza un recorrido histórico al respecto y concluye que, considerando a la legitimación de conocimientos como tarea de la escuela y a la generación de conocimientos como tarea de la 
universidad, resulta crucial la articulación escuela - universidad, para que la población pueda comprender, analizar y transformar la realidad.

Silvia Morelli (Facultad de Humanidades y Artes), en el capítulo titulado "Currículum, alfabetización científica y cambio tecnológico. Desafíos para el siglo XXI" (pp. 77-89), identifica tres aspectos clave para afrontar los desafíos educativos de estos tiempos: la alfabetización científico-tecnológica y la enseñanza de las ciencias, contemplando aquí capacidades y aptitudes en las prácticas científicas, el carácter interdisciplinario de los saberes involucrados, la proximidad de estos saberes hacia campos teóricos trascendiendo lo técnicoinstrumental y el reconocimiento del contexto social y cultural de las tecnologías; el cambio tecnológico, donde realiza una lectura sociológica de las tecnologías y el currículum desde las posibilidades de acceso de la población; la educación ambiental, la educación obligatoria y la educación de la ciudadanía, como intrínsecamente relacionadas al pensar la sustentabilidad y el desarrollo.

Estela Álvarez (Secretaría de Ciencia y Tecnología), en el capítulo titulado "Educación, ciencia y tecnología" (pp. 91-99), comenta las acciones que se vienen desarrollando en el Programa Iberoamericano IBERCIMA, dedicado a la enseñanza de las Ciencias y la Matemática en el nivel medio. Entre ellas reconoce al análisis de: planes y programas de estudio, formación inicial y capacitación permanente de los profesores, materiales e instrumentos de apoyo, inventarios de recursos materiales y humanos con que se cuenta, actividades extracurriculares que favorezcan la movilización y motivación de docentes y alumnos. Reconoce a la alfabetización científica como exigencia urgente, y sostiene que se está invirtiendo en ello, para el desarrollo inmediato.

Elisa Petrone (Facultad de Ciencias Exactas, Ingeniería y Agrimensura), en el capítulo titulado "Aprender Matemática: en la variedad está el gusto" (pp. 101-114), comienza por preguntarse para qué enseñar Matemática y apunta la respuesta hacia el aprendizaje de los alumnos, con cuotas de creatividad, compromiso y efectividad, con el objetivo de formar personas pensantes, que sepan abordar la resolución de diversas situaciones problemáticas y que disfruten al hacerlo. Para lograr esto, identifica como fundamental a la actitud del docente y la pluralidad de variadas actividades que se propongan a los alumnos. En esta variedad reconoce la diversidad de contenidos, de lenguajes y de procesos de validación, así como de recursos empleados y de habilidades puestas en juego. Sugiere que esta variedad se distribuya a lo largo de un ciclo lectivo y no necesariamente en cada tema que se trabaje.

Celia Machado (Facultad de Ciencias Bioquímicas y Farmacéuticas), en el capítulo titulado "El enfoque CTS en la enseñanza de las ciencias experimentales" (pp. 115-130), admite la preocupación por la utilización de los medios informativos sobre las actividades científico-tecnológicas para facilitarle a las personas su inserción en un mundo complejo, desconocido y cambiante. Para trabajar al respecto propone el enfoque CTS (ciencia-tecnología-sociedad) en 
la enseñanza de las ciencias, entre cuyos objetivos destaca: incrementar la comprensión de los conocimientos científico-tecnológicos, potenciar los valores de la ciencia y la tecnología y desarrollar las capacidades de los estudiantes. Propone para ello el desarrollo de unidades didácticas en contexto y ejemplifica con las bebidas energizantes/estimulantes.

Marta Massa (Facultad de Ciencias Exactas, Ingeniería y Agrimensura), en el capítulo titulado "La comprensión del discurso del libro de texto como contenido de enseñanza" (pp. 131-146), menciona que la forma en que el libro presenta los contenidos, las cuestiones que plantea, el lenguaje en sus distintas modalidades que utiliza, el modo de provocar la reflexión y el razonamiento, se constituyen en elementos básicos para producir ciertas visiones (integradas o parcializadas) de conocimiento en los estudiantes. Plantea que la elección de este tipo de material es una decisión curricular importante para el profesor porque supone definir un enfoque didáctico para su uso en el aula. Le atribuye al texto una función comunicativa, que se puede presentar en distintas formas: evocación, información, definición, descripción, aclaración, ejemplificación, comparación, apelación a la analogía, problematización, explicación, interpretación, apelación a la metáfora y aplicación, siendo el docente el responsable de ponerlas efectivamente en práctica.

Lucrecia Dasso y Sergio Cavaglia (Secretaría de Extensión Universitaria), en el capítulo titulado "La extensión en la universidad. Una herramienta para una estrategia de desarrollo" (pp. 147-157) conciben a la extensión universitaria como una dimensión más del proceso educativo, trascendiendo el asistencialismo o la prestación de servicios, y que se articula transversalmente con las restantes funciones de la universidad. Desde la Secretaría en que se desempeñan, tienen el objetivo de alentar y promover acciones transformadoras y generadoras de aptitudes con alcances en los actores del medio social, la comunidad científica y el sector productivo. Apuntan al mismo desde cuatro pilares de desarrollo: productivo y medio ambiental; sociocomunitario y sanitario; educativo y cultural; universidad de gestión pública e infraestructura.

Se coincide con Liliana Sanjurjo (Facultad de Humanidades y Artes), quien realiza el prólogo del libro (pp. 11-15), al reconocer a esta obra como una forma de concretar ciertas funciones de la universidad: producir conocimiento, socializar producciones, contribuir en la resolución de problemáticas del contexto, colaborar en el desarrollo y mejora de otros niveles educativos. 